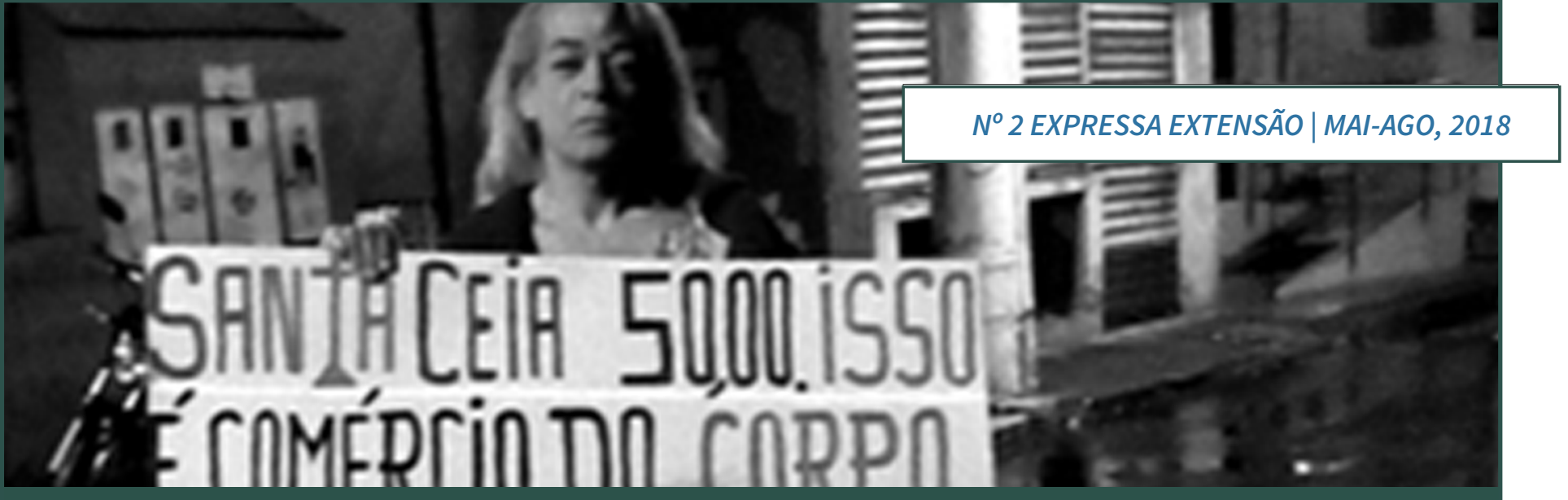

\title{
O OLHAR ENGAJADO E O CUIDADO COM O SER: DESENVOLVENDO A PRÁTICA FOTOGRÁFICA E OS SENTIDOS POR MEIO DA JORNADA DE EXTENSÃO E CULTURA UNIVERSITÁRIA
}

THE ENGAGED LOOK AND CARE THE BEING: DEVELOPING THE PHOTOGRAPHIC PRACTICE AND THE SENSES THROUGH THE SEMINAR OF EXTENSION AND UNIVERSITY CULTURE

Vitorino Fontenele Freire ${ }^{1}$ Marcelo Calderari Miguel ${ }^{2}$

\section{RESUMO}

A estética fotográfica enquadra-se na possibilidade de desenvolvimento de linguagem para os que dela se utilizem. Logo esse resgate memorial é fruto do aprendizado da oficina "Olhar Fotográfico" da Jornada Integrada de Extensão e Cultura da Pró-Reitoria de Extensão - Área Temática Cultura e Área Afim de Comunicação Social, sendo a Linha de Extensão a questão da Produção Cultural e Artística na Área de Fotografia, Cinema e Vídeo. Com a aprendizagem dessa oficina realizamos o presente ensaio fotoetnográfico trazendo em pauta o tema da liberdade de consciência e o cuidado e visibilidade do ser. Ao desenvolver o chamado 'olhar fotográfico' expande-se as possibilidades criativas para reconhecer e explorar as melhores alternativas de composição que cada ambiente pode proporcionar. O produto dessa atividade extensionista vai além do tema retratado e da pessoa acolhida nessas fotografias; muitas outras esferas sociais e pessoais a oficina desperta - tal como empatia e respeito como qualidades inerentemente humanas. Observa-se que as ilustrações deste ensaio reproduzem mais que o superficial incrustado; muitas outras associações e complexidades a fotografia desperta, e gera novas possibilidades de formação e debate sobre o campo da arte, seu ensino e seus modos de presença na sociedade.

Palavras-chave: Fotografia. Ação educativa. Imagem. Produção cultural.

\footnotetext{
${ }^{1}$ Vitorino Fontenele Freire - UFES - Brasil - Mestrando em Ciências Sociais pela Universidade Federal do Espirito Santo - UFES. Licenciado em Ciências Sociais, Bacharel em Ciências Contabeis e em Ciências Sociais pela Universidade Federal do Espírito Santo. Pesquisador de Povos e Comunidades Tradicionais (PCTs), vitorinobb@ yahoo.com.br; ${ }^{2}$ Marcelo Calderari Miguel - UFES - Brasil - Especialista em Educação Científica pela Universidade Federal de Minas Gerais - UFMG; Bacharel em Bibliotecomia pela Universidade Federal do Espirito Santo - UFES, marcelocalderari@yahoo.com.br
} 


\section{ABSTRACT}

The photographic aesthetic is framed in the possibility of developing language for those who use it. Soon this memorial rescue is the result of the learning of the photography workshop "Olhar Fotográfico" of the Integrated Extension and Culture Seminar of the Pro-Deanship Of Extension at Ufes - Thematic Area of Culture and Social Communication, the Extension Line being the issue of Cultural Production and Artistic in the Area of photography, cinema and video. With the learning of this workshop we carry out the present photoetnographic essay bringing the issue of freedom of conscience and the care and visibility of being into the agenda. Developing the so-called 'photographic look' expands the creative possibilities to recognize and explore the best compositional alternatives that each environment can provide. The product of this extension activity goes beyond the theme portrayed and the person welcomed in these photographs; many other social and personal spheres the workshop awakens - such as empathy and respect as inherently human qualities. It has been observed that the illustrations of this essay reproduce more than the embedded surface, many other associations and complexities an awakened photograph, and it generates new possibilities of formation and debate on the field of art, its teaching and its modes of presence in society.

Keywords: Photography. Educational action. Image. Cultural production.

\section{JORNADA DE EXTENSÃO E CULTURA}

A arte é um recurso potente para desenvolver a sensibilidade e senso crítico, possibilitando novos olhares e um novo modo de perceber o mundo, relacionando questões históricas e sociais ao contexto atual. E o apoio da Pró-Reitoria de Extensão tem sido fundamental para a inserção do projeto 'Oficina de Fotografia - Olhar Fotográfico' na $5^{a}$ Jornada ${ }^{3}$ Integrada de Extensão e Cultura da Universidade Federal do Espírito Santo (UFES), acreditando em sua potência para o desenvolvimento da sensibilidade e senso crítico para questões relacionadas ao cuidado com a vida.

Durante a oficina, o aluno será estimulado a desenvolver o seu próprio olhar fotográfico considerando, antes de cada clique, os vários elementos estéticos que compõem uma fotografia, tais como: proposta visual; [...] contraste; linhas retas, diagonais, curvas e imaginárias; simetria e enquadramento. O objetivo da oficina é fazer com que o aluno pense o ato de fotografar como um processo dinâmico e criativo, que começa muito antes de cada clique e se estende até as inúmeras possibilidades que a arte pode lhe oferecer (SIEX, 2017, p. 1).

\footnotetext{
3 Evento com o tema "Por uma extensão sustentável" visa divulgar as ações de extensão da universidadee discutir formas de tornar as açõese projetos afinados a uma sociedade sustentável.
} 
Esse ensaio é 'produto' da oficina "Olhar Fotográfico", cujo escopo é demonstrar que a ciência está nas linhas, cores, pinturas e fotografias -"as quais são forma de registro reflexivo e sensibilizante" (SIEX, 2017, p. 1). E de acordo com Goldblatt (1961), o gênio renascentista Leonardo Da Vinci (1452-1519) considerava o olho humano como 'janela da alma' e o principal instrumento para o artista e o cientista poder contemplar as magníficas, abundantes e infinitas obras da natureza.

\section{FOTO E ENFOQUE: EIS A LIBERDADE DE CRENÇA E DE CONSCIÊNCIA}

Este ensaio visual tem como foco a análise do depoimento e fotos de protesto, através do levantamento de cartazes, relativos a uma mulher transexual, antropônimo Mel, 50 anos, que demonstra sua insatisfação com a decisão de um pastor de uma igreja de Vitória (na capital Espírito-santense) que proibiu a entrada de transexuais na sua igreja pentecostal.

Ressaltamos que a imagem fotográfica "sempre designa coisas e estados de coisas, exprime simultaneamente eventos" (ROUILLÉ, 2009, p. 204). Assim, a seguir transcrevemos trechos do depoimento da transexual concebido durante a abordagem fotoetnográfica que compõem esse ensaio visual.

'Mel' é cabeleireira e reportou que o problema com o pastor existe esde 2016. Em sua 'idas à igreja' teve uma experiência espiritual - a "manifestação" foi intuída pelo pastor como "algo demoníaco". A transexual alega que sofre com a "homofobia sexual e violência do referido pastor", e assim, segue em frente da Igreja em protesto 'semissilencioso' nesses últimos dois anos.

Convém lembrar que o "sofrimento ético" retrata a vivência cotidiana das questões sociais dominantes em cada época histórica, notadamente, a "dor" que surge da situação social de ser tratado como inferior, de pouco valor, escória social (SAWAIA, 2006). 
Figura 1 - Ver não é simplesmente ver; não é simplesmente perceber com os olhos!

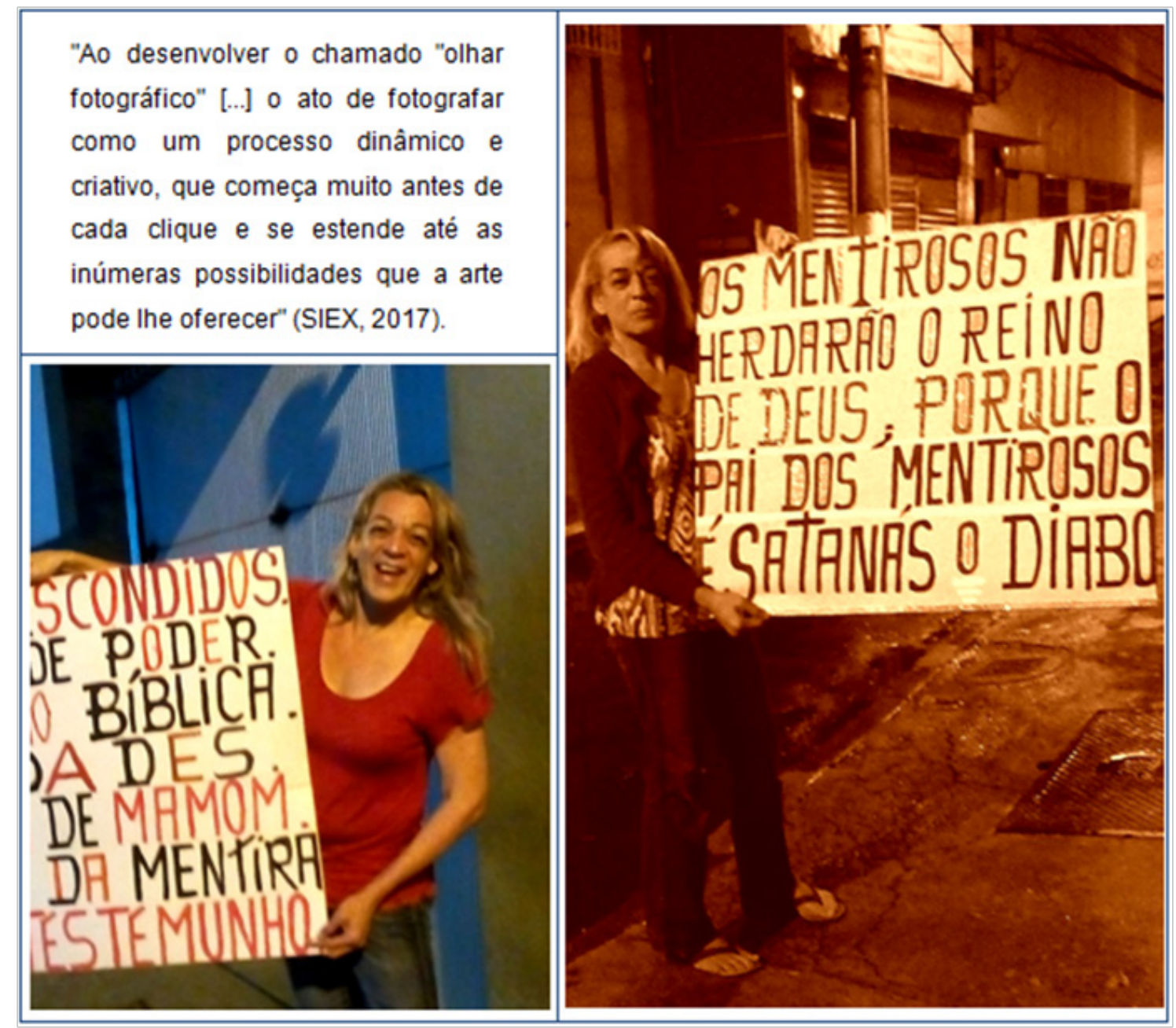

Fonte: elaborado pelos autores, Centro, Vitória - Espírito Santo (2017)

E Mel questiona a função da Igreja: "deveria pregar o amor" e, no entanto, "incita a violência, como pode?" Querem que "eu corte meu cabelo, que use roupas de homem, como pode?" Só porque "sou homossexual ou transexual sou proibida de entrar?" Uma igreja protestante "pode ferir [minha natureza humana?]" São 'várias perguntas' e mensagens em cartazes que não querem calar. 
Figura 2 - O ser requer cuidado: (re)descoberta e (re)encontro ou exclusão?

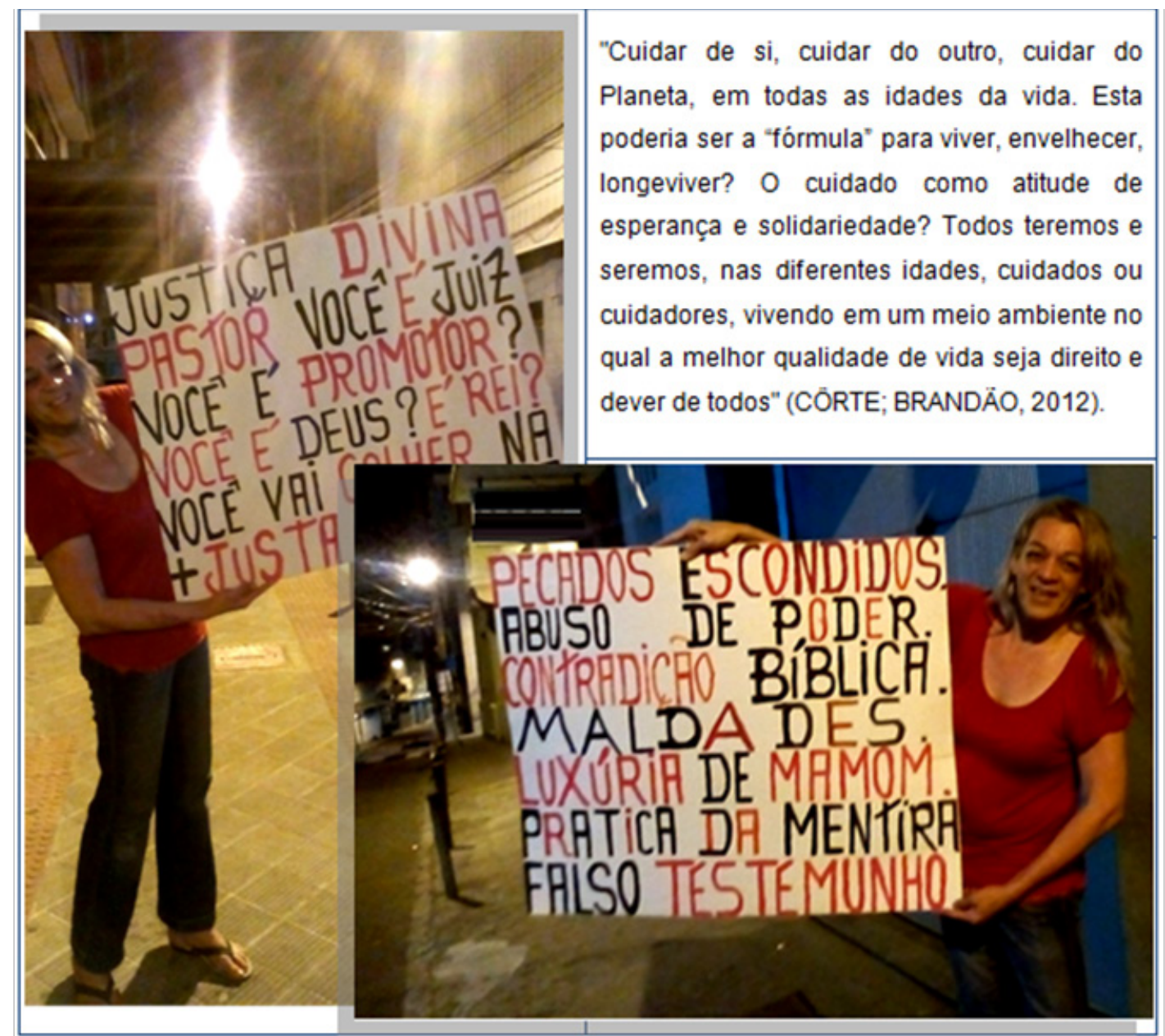

Fonte: elaborado pelos autores, Vitória - ES (2017).

Faz parte do exercício de debate democrático e está amparado pela Carta Magna $^{4}$ a liberdade de opinião e expressão o direito de criticar uma determinada religião ${ }^{5}$, desde que não haja desrespeito a quem for direcionada a crítica. E nossa Constituição Federal de 1988 assegura que a liberdade de consciência e de

\footnotetext{
${ }^{4}$ E Com relação a prática da religião no Brasil a Constituição Federal de 1988 assegura alguns direitos, tais como: a liberdade de consciência e de crença, sendo assegurado o livre exercício dos cultos religiosos e garantida, na forma da lei, a proteção aos locais de culto e a suas liturgias (Art. $5^{\circ}$, inciso $\mathrm{VI}$ ) e, sendo vedado embaraça-lhe o funcionamento (Art. 19 $9^{\circ}$ inciso I). ${ }^{5}$ As ciências modernas têm suas raízes na tradição judaico-cristã, mas divergem em seus fundamentos. A ciência tem como fundamento os paradigmas científicos que vão se alterando ao longo do tempo e que se apoiam na razão, enquanto a religião tem como firme fundamento a fé que possuem um forte apoio em seus dogmas religiosos.
} 
crença é inviolável e salvaguarda ao cidadão o direito de expressar e viver sua religiosidade

Depreende-se assim, que a significância da tolerância em todas as religiões não deve constituir impedimento para que haja um relacionamento fraterno, respeitoso e solidário entre os cidadãos. Portanto, todos devem ter isonomia de tratamento perante a lei, independente da sua orientação religiosa.

Figura 3 - Inclusão social: Lutar ou a arte de simplesmente viver?

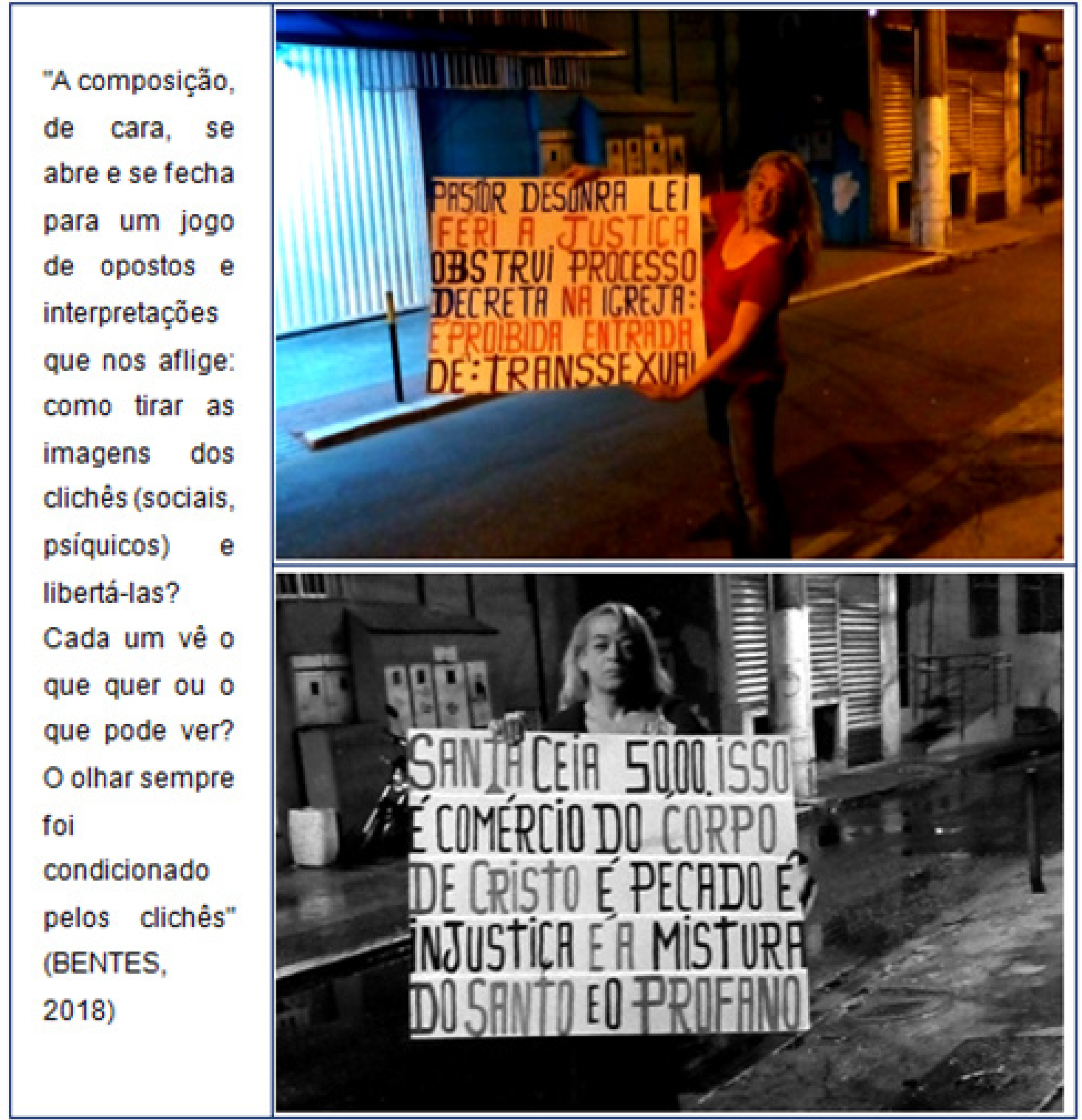

Fonte: elaborado pelos autores, Vitória (2017). 
Em suma, compreende-se que "a imagem não fala por si só; é necessário que as perguntas sejam feitas" (MAUAD, 2005, p. 144). Entende-se ser desnecessário o embate entre a cidadã Mel e o pastor considerando que se o(a) cidadão(ã) que não for bem aceito(a) em determinada 'crença religiosa' em decorrência dos 'dogmas dessa'o mais conveniente é procurar outro credo religioso que o(a) aceite - considerando a grande diversidade de entidades religiosas (instituições autodenominadas de 'igrejas inclusivas') existentes no Brasil.

Numa comparação grosseira a religião é como se fosse um contrato de adesão. O cidadão vai poder aderir a uma religião se a sua fé, seus valores, sua cosmologia se coadunarem notadamente com os dogmas religiosos e a interpretação do Livro Sagrado adotados por essa Igreja. Considerando as inúmeras crenças religiosas não fica tão difícil o(a) cidadão(ã) identificar um credo religioso que seja aderente aos seus valores. A Igreja só aceita os fiéis que concordarem viver de acordo com seus dogmas.

Além do mais, o que se vê vai "além do aparente, essa visão pode ser sinestésica, ela nos suscita histórias afetivas, referências e escolhas. Podemos olhar como podemos escolher não olhar para uma dada realidade" (AGUIAR; MATOS, 2017, p. 3). Silva e Cerqueira-Santos $(2014$, p. 36) reportam que muitas vezes a história de vida dos(as) travestis e transexuais mostra que não há sentimento de pertencimento dessas pessoas ao "seu grupo [comunidade $L G B T Q I+{ }^{6}$ ], já que a travestilidade e a transexualidade não é por diversas vezes socialmente aceita" e isso resulta em carências.

A fotografia, na rota lexical, expressa um ponto de vista. Nela cabe o espanto, o acolhimento e há um dado olhar, mas diversos são possíveis ou transformados:" afinal, cada um vê o que quer ou o que pode ver?"(BENTES, 2018, p.1). E segundo esse projeto de extensão "uma fotografia nos olha de volta", e nós a atravessamos em infindáveis olhares cada vez que revemos ou a revisitamos.

\footnotetext{
${ }^{6}$ A sigla aqui usada será a LGBTQI+ (lésbicas, gays, bissexuais, transgêneros, queers, intersexuais e o sinal de '+' adicionado no sentido de representar qualquer outra pessoa que não seja coberta pelas seis outras iniciais), pela importância que os autores veem na inclusão de outras sexualidades e gêneros na sigla que representa tantos grupos e movimentos.
} 


\section{REFERÊNCIAS}

AGUIAR, Ana Lídia; MATOS, Fernanda. Aprendendo a "olhar": o uso de imagens em sala de aula. Revista Pensata, Guarulhos, v. 6, n. 1, p. 1-16, dez. 2017. Disponível em: <http://www2.unifesp.br/revistas/pensata/?attachment_id=1027>. Acesso em: 13 jan. 2018.

BENTES, Ivana. 0 que vemos e o que nos olha. São Paulo: Grupo Cult, 2018. Disponível em: <https://revistacult.uol.com.br/home/ivana-bentes-o-que-vemos-eo-que-nos-olha/>. Acesso em: 13 jan. 2018.

CÔRTE, Beltrina;BRANDÃO, Vera. Cuidar de si, cuidar do outro, cuidar do planeta. Revista Portal de Divulgação [do Envelhecimento], São Paulo, n.27, p. 1-6, nov. 2012. Disponível em: <http://www.portaldoenvelhecimento.com/revista-nova/index.php/revistaportal/article/viewFile/301/301 >. Acesso em: 11 jan. 2018.

GOLDBLATT, Maurice Henry. Leonardo da Vinci: a newly identified head of Leda:a newly identified design for a standing Leda how the paintinga of salaino were identified. -. New York: Citadel, 1961.

MAUAD, Ana Maria. Na mira do olhar: um exercício de análise da fotografia nas revistas ilustradas cariocas, na primeira metade do século XX. An. Mus. Paul., São Paulo, v. 13, n. 1, p. 133-174, jun. 2005. Disponível em: <http://dx.doi.org/10.1590/ S0101-47142005000100005>. Acesso em: 13 jan. 2018.

ROUILLÉ, André. A fotografia: entre documento e arte contemporânea. São Paulo: Senac, 2009.

SAWAIA, BaderBurihan. As artimanhas da exclusão: análise psicossocial e ética da desigualdade social. 6. ed. Petrópolis: Vozes, 2006.

SIEX [Sistema de Informações de Extensão da Ufes]. Oficina de Fotografia: "Olhar Fotográfico" na 5. Jornada Integrada de Extensão e Cultura. Vitória, out. 2017. Disponível em: <http://siex1.ufes.br/siex/AuditarCurso.do?id=11380>. Acesso em: 19 jan. 2018.

SILVA, Bruno de Brito; CERQUEIRA-SANTOS, Elder. Apoio e suporte social na identidade social de travestis, transexuais e transgêneros. Rev. SPAGESP, Ribeirão Preto, v. 15, n. 2, p. 27-44, dez. 2014.Disponível em: <http://pepsic.bvsalud. org/scielo.php?script=sci_arttext\&pid=S1677-29702014000200004 >. Acesso em:7 abr. 2018.

Data de recebimento: 19 de fevereiro de 2018.

Data de aceite para publicação: 13 de abril de 2018. 